\title{
STELLA MARIS: Stellar Marine Refractive Imaging Sensor
}

\author{
Marina Alterman, Yohay Swirski and Yoav. Y. Schechner \\ Dept. of Electrical Engineering, \\ Technion - Israel Institute of Technology, Haifa, Israel \\ amarina@tx.technion.ac.il,yohays@tx.technion.ac.il,yoav@ee.technion.ac.il
}

\begin{abstract}
Viewing an airborne scene from a submerged camera creates a virtual periscope, avoiding the saliency of a real maritime periscope. Random waves in the water-air interface severely distort the view, by refraction. We show a way to handle this. The distortion can be significantly countered, based on an estimate of the wavy water interface, at the instant of imaging. We obtain the interface estimate per frame, by a submerged sensor that simultaneously images the refracted Sun through an array of submerged pinholes. Our use of a stellar cue for estimation (and then correction) of the refractive field has analogy to ground-based astronomy. In astronomy, a guide star is imaged by a ShackHartmann sensor, for an estimate of the random refractive field created by atmospheric turbulence. In astronomy, this principle is used for countering blur, mainly by adaptive optics, while we use it for compensating distortions created by water waves. We introduce this novel concept for enabling a virtual periscope, demonstrate it, and analyze some of its limitations.
\end{abstract}

\section{Introduction}

Viewing an airborne scene from a submerged camera creates a virtual periscope. Contrary to a true periscope, a virtual one is completely submerged, and does not draw attention. Images taken this way suffer severe refractive distortions, even if the water-air interface (WAI) is flat $[2,35]$. For distant objects, distortions attributed to flat-water can be countered by optical and computational methods [44]. Random waves of the WAI greatly exacerbate the distortions. They are much more severe than distortions created by atmospheric turbulence $[15,21,41,54]$, due to the sharp difference of water and air refractive indices. If the WAI shape can be estimated, distortions attributed to the waves can be countered. Recovering the WAI in a virtual-periscope setting is very challenging. A virtual periscope should avoid attention outside, which overrules placing a known artificial calibration object beyond the WAI [7, 24, 25, 52] (in air). For the same reason, active excitation or structured
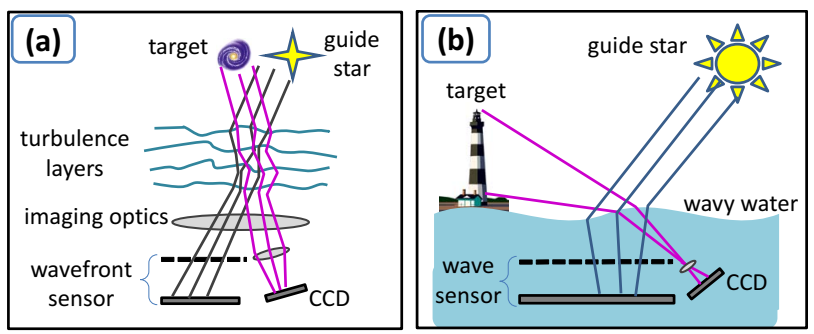

Figure 1. (a) Astronomy suffers from random refractive disturbances caused by atmospheric turbulence. The disturbance is estimated based on a wavefront-sensor that measures a projection of a guide star. (b) A submerged virtual periscope suffers from random refractive disturbances caused by a wavy water-air interface. The disturbance is estimated based on a surface-slope-sensor (wave sensor). Here the guide star is the Sun. In both cases, the disturbance estimate enables its partial compensation.

light $[6,12,18,31,34,38,51,53]$ is inhibited. Hence, we need passive sensing of the WAI.

Passive recovery of the WAI for a virtual periscope ${ }^{1}$ was theorized, by possible analysis of the radiance near the edge of Snell's window [22], or sky polarization [40]. We, however, take a different approach, that yields a practical implementation. We observe an analogy between our problem and ground-based astronomy (Fig. 1). Telescopes view celestial scenes through a random refracting medium: the turbulent atmosphere. Astronomical image degradation can be largely countered by adaptive optics and computational steps, based on an estimate of the refractive disturbance. This estimate is obtained by measuring a known guide star using, typically, a Shack-Hartmann wavefront sensor.

In analogy, images taken by a submerged virtual periscope are degraded by the random refractive WAI, and estimation of this refractive disturbance would facilitate compensation. The analogy of the problem points to our solution: the WAI can be estimated by measuring a known guide star through the WAI, simultaneously with viewing

\footnotetext{
${ }^{1}$ Many passive methods for recovering transparent surfaces [13, 37] are often incompatible with a virtual periscope. Specifically $[14,39]$, rely on reflection of the sun or sky by the WAI, rather than refraction.
} 


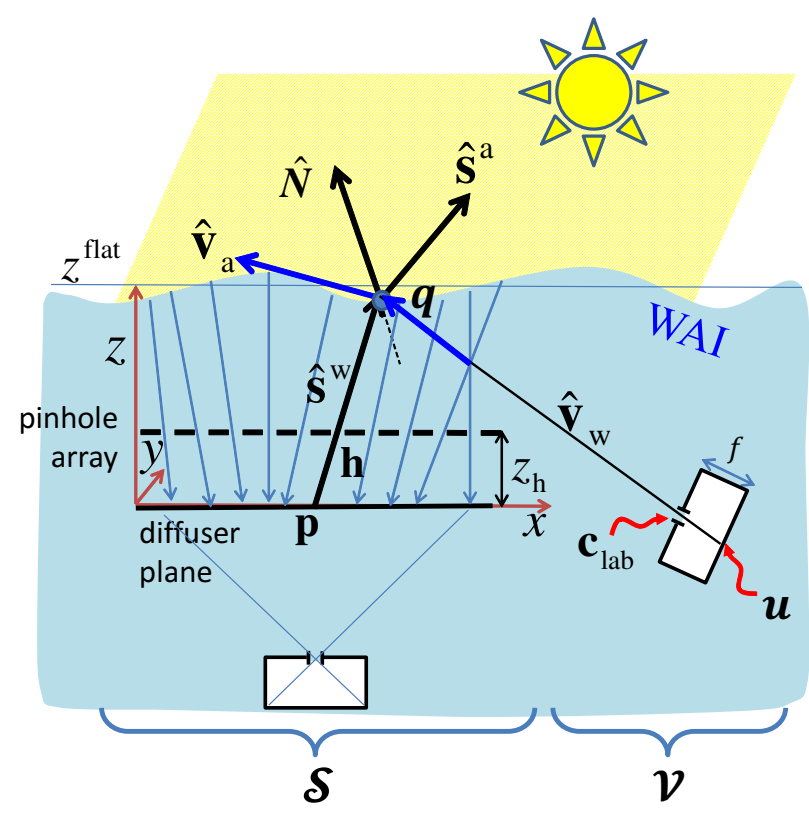

Figure 2. Geometry of a stellar marine refractive imaging sensor (STELLA MARIS), comprising a slope-sensor $\mathcal{S}$, a viewing camera $\mathcal{V}$, and the Sun pointed by airborne vector $\hat{\mathbf{s}}^{\mathrm{a}}$.

the scene of interest. Our stellar guide is obvious: the Sun. ${ }^{2}$ It is observed over an array of submerged locations, in analogy to a Shack-Hartmann astronomical sensor. Our STELLAr MArine Refractive Imaging Sensor (STELLA MARIS) resembles a light-field or integral-imaging camera $[4,11,19,28]$. The measured stellar light field indicates the WAI deformation across the field of view. ${ }^{3}$ This enables significant reduction of distortion, using physicsbased geometric computations, per frame. We develop this idea, demonstrate it, and analyze some of its limitations.

\section{View Through a Water-Air Interface}

Consider Fig. 2. Component $\mathcal{V}$ of the imaging sensor views the airborne scene through the wavy WAI. Suppose for the moment that the WAI surface is known: we shall deal with its estimation in Sec. 3. Given the WAI, here we analyze distortion and its compensation.

An internal coordinate system $\mathcal{V}$ consists of its optical axis and the lateral pixel coordinates of the image plane. The origin of $\mathcal{V}$ is at the center of its projection. The optical axis of $\mathcal{V}$ intersects the image plane at image-plane location c. The focal length of $\mathcal{V}$ is $f$. In this coordinate frame, the three-dimensional (3D) location of a detector element (pixel) $\mathbf{u}$ is $\mathbf{u}^{\text {cam }}=\left[(\mathbf{u}-\mathbf{c})^{\mathrm{T}},-f\right]^{\mathrm{T}}$, where $\mathrm{T}$ denotes trans-

\footnotetext{
${ }^{2}$ Sun rays that refract through the random WAI create flickering caustics. In [36], the disturbing random pattern was attenuated, while in [45] it was exploited to solve the stereo correspondence problem.

${ }^{3}$ Active illumination from below the water, projecting upwards to an airborne camera, was used to study waves in [31].
}

position. Relative to the global coordinate system, the pose of $\mathcal{V}$ is defined by a rotation matrix $\mathbf{R}$ and a translation vector $\mathbf{t}$. In the global (lab) coordinate system, the 3D position of the detector element is

$$
\mathbf{u}_{\text {lab }}=\mathbf{R}^{\mathrm{T}}\left(\mathbf{u}^{\text {cam }}-\mathbf{t}\right) .
$$

Setting $\mathbf{u}^{\text {cam }}=0$ in Eq. (1), the origin of $\mathcal{V}$ in the global coordinate system is $\mathbf{c}_{\text {lab }}=-\mathbf{R}^{\mathrm{T}} \mathbf{t}$.

We back-project a ray from $\mathbf{u}_{\text {lab }}$ through $\mathbf{c}_{\text {lab }}$. In a perspective system the back-projected ray propagates in water as

$$
\mathcal{R}^{\mathrm{w}}(\mathbf{u}) \equiv\left\{\mathbf{c}_{\mathrm{lab}}+\hat{\mathbf{v}}_{\mathrm{w}} l\right\}_{\forall l>0},
$$

where the ray direction vector is

$$
\hat{\mathbf{v}}_{\mathrm{w}}=\left(\mathbf{u}_{\mathrm{lab}}-\mathbf{c}_{\mathrm{lab}}\right) /\left\|\mathbf{u}_{\mathrm{lab}}-\mathbf{c}_{\mathrm{lab}}\right\|
$$

and $l$ parameterizes longitudinal propagation along the ray. The backprojected ray intersects the WAI at

$$
\mathbf{q}(\mathbf{u})=\mathrm{WAI} \cap \mathcal{R}^{\mathrm{w}}(\mathbf{u}),
$$

where the WAI normal is $\hat{\mathbf{N}}$. Based on a vector form of Snell's law, the airborne viewing direction vector is

$$
\hat{\mathbf{v}}_{\mathrm{a}}=n \hat{\mathbf{v}}_{\mathrm{w}}+\hat{\mathbf{N}}\left[\sqrt{1-n^{2}+n^{2}\left(\hat{\mathbf{v}}_{\mathrm{w}} \cdot \hat{\mathbf{N}}\right)^{2}}-n \hat{\mathbf{v}}_{\mathrm{w}} \cdot \hat{\mathbf{N}}\right] \text {. }
$$

Here $n$ is the refractive index of water, where $n \approx 1.33$. For an object at an effectively infinite distance, $\hat{\mathbf{v}}_{\mathrm{a}}$ is the undistorted coordinate vector pointing at the object.

Even if $\mathcal{V}$ has a single viewpoint, refraction by the wavy WAI disperses the effective viewpoints to a wide locus. Hence, for a nearby object, different parts are viewed from different effective viewpoints. Different 3D object parts can be occluded by other object parts, in different frames, as the viewpoints change. This bars complete un-distortion [49]. Partial correction can be achieved using some approximate assumptions, e.g., that the object is flat, and at known distance. Suppose the object lies on a plane $\Pi^{\text {object }}$. The airborne back-projected ray is $\mathcal{R}^{\mathrm{a}}(\mathbf{u}) \equiv\left\{\mathbf{q}+\hat{\mathbf{v}}_{\mathrm{a}} l\right\}_{\forall l>0}$. The object projecting to image pixel $\mathbf{u}$ is at $3 \mathrm{D}$ location $\mathbf{a}=\Pi^{\text {object }} \cap \mathcal{R}^{\mathrm{a}}(\mathbf{u})$. Point a can then be computationally projected through $\mathcal{V}$, or any other system.

\section{Guide Star Refraction Estimation}

Ground-based astronomy is affected by random refraction in the air [32], across the telescope aperture and time (Fig. 1). To partly correct for this, telescopes use wave-front sensors [30]. Specifically, the Shack-Hartmann sensor [31] resembles a light-field camera, or integral-imager [5, 43]. It images a distant known guide star through an array of lenslets or pinholes. From deviations of the resulting star imagelets, the instantaneous refractive disturbance is spatially sampled and estimated. Then, this disturbance can be countered, e.g., using adaptive optics [42]. 
We take a similar approach to sense the refractive disturbance. In our case, this disturbance stems from a non-flat WAI slope, which in unknown and varies spatiotemporally. Our guide star is the Sun. The WAI-slope sensor includes a horizontal array of pinholes (Fig. 2), in analogy to a ShackHartmann sensor. Sun rays project to the focal plane of the pinholes, forming a small distorted image of the Sun, per pinhole. The lateral displacement of the solar image-lets varies with the local slope of the WAI. Thus, from the acquired image data, the WAI is estimated.

The system has to be sufficiently deep, to ensure its submersion during waves. At the same time, we let $\mathcal{V}$ have somewhat widefield imaging. Hence, the field of view crosses a wide area $\mathcal{A}$ of the WAI, measuring decimeters across. Instead of deploying an expensive detector array or lens aperture of this size, we take an indirect way to capture the array of solar image-lets (Fig. 2). Rays passing the pinhole array project to a wide planar diffuser, parallel to the plane of pinholes. The diffuser is imaged by a camera. To sum up, we devise a stelar-guided slope-sensor $\mathcal{S}$, comprising a pinhole array, a diffuser, and a standard camera.

\subsection{Stellar Sampling of WAI Normals}

A sunray refracts by the WAI, then passes through a pinhole at 3D location $\mathbf{h}$, and irradiates a spot on the diffuser (Fig 2), at 3D location $\mathbf{p}$. The unit vector $\hat{\mathbf{s}}^{\mathrm{w}}$ is the direction of a back-projected sunray in the water, from $\mathbf{p}$ via $\mathbf{h}$,

$$
\mathbf{s}^{\mathrm{w}}=\mathbf{h}-\mathbf{p} ; \quad \hat{\mathbf{s}}^{\mathrm{w}}=\mathbf{s}^{\mathrm{w}} /\left\|\mathbf{s}^{\mathrm{w}}\right\|_{2} .
$$

In air, the unit vector pointing to the Sun is $\hat{\mathbf{s}}^{\mathrm{a}}$. Using a different vector form of Snell's law [16] at the water interface,

$$
\hat{\mathbf{s}}^{\mathrm{a}} \times \hat{\mathbf{N}}=n \hat{\mathbf{s}}^{\mathrm{w}} \times \hat{\mathbf{N}} \text {. }
$$

Here $x$ is the cross product. In the global coordinate system, the horizontal $(x, y)$ coordinates correspond to the pinhole array axes, and $z$ denotes height above the diffuser plane. The pinhole array is at distance $z_{h}$ above the diffuser. Using the axial components of the vectors $\hat{\mathbf{s}}^{\mathrm{a}}, \hat{\mathbf{s}}^{\mathrm{w}}$ and $\hat{\mathbf{N}}$, Eq. (7) can be phrased in matrix form,

$$
\mathbf{A N}=0,
$$

where

$$
\mathbf{A}=\left(\begin{array}{ccc}
0 & -s_{z}^{\mathrm{a}}+n s_{z}^{\mathrm{w}} & s_{y}^{\mathrm{a}}-n s_{y}^{\mathrm{w}} \\
s_{z}^{\mathrm{a}}-n s_{z}^{\mathrm{w}} & 0 & -s_{x}^{\mathrm{a}}+n s_{x}^{\mathrm{w}} \\
-s_{y}^{\mathrm{a}}+n s_{y}^{\mathrm{w}} & s_{x}^{\mathrm{a}}-n s_{x}^{\mathrm{w}} & 0
\end{array}\right) .
$$

The vector pointing to the Sun in air, $\hat{\mathbf{s}}^{\mathrm{a}}$, is always known, given the time of image acquisition, geographic location, and compass azimuth of the global coordinate system. Moreover, the vector $\hat{\mathbf{s}}^{\mathrm{w}}$ is extracted from the image data (6). Hence, the matrix $\mathbf{A}$ is known. The WAI normal $\hat{\mathbf{N}}$ is estimated by solving Eq. (8): it is the null subspace of $\mathbf{A}$. This process is repeated for each pinhole, indexed $k$, and located at $\mathbf{h}_{k}$. It yields a set of sampled vectors $\left\{\hat{\mathbf{N}}_{k}\right\}$.

\subsection{Where are the WAI-normal samples?}

Let sensor $\mathcal{S}$ be periodic: the distance between nearby pinholes is uniform. Nevertheless, the system samples the normal field $\hat{\mathbf{N}}(x, y)$ non-periodically. As in Eqs. $(2,4)$, the in-water sunray back-projected from pinhole $k$ is

$$
\mathcal{R}_{k}^{\mathrm{w}} \equiv\left\{\mathbf{h}_{k}+\hat{\mathbf{s}}^{\mathrm{w}} l\right\}_{\forall l>0},
$$

This backprojected ray intersects the WAI at

$$
\mathbf{q}_{k}=\mathrm{WAI} \cap \mathcal{R}_{k}^{\mathrm{w}} .
$$

The sample normal $\hat{\mathbf{N}}$, estimated in Sec. 3.1, corresponds to $3 \mathrm{D}$ point $\mathbf{q}_{k}$. However, $\mathbf{q}_{k}$ derived in Eq. (11) is unknown, since the WAI is unknown. For instance, if we computationally bias the height of the wavy WAI above the sensor, an oblique $\mathcal{R}_{k}^{\mathrm{w}}$ would intersect the WAI at a laterally shifted location. Moreover, due to WAI curvature, the direction $\hat{\mathbf{s}}^{\mathrm{w}}$ varies for different pinholes. Thus Eq. (11) does not tightly follow the periodicity of the pinholes.

The set $\left\{\mathbf{q}_{k}\right\}$ is quasi-periodic, having a perturbation to periodicity. To operate with these uncertainties, approximate assumptions are made. We explored various constraints with increasing complexity, and settled on the following. For solving Eq. (11), we artificially set the WAI to be flat, at altitude $z^{\text {flat }}$ above the diffuser plane. This value is the time-averaged underwater depth of the diffuser. The value of $z^{\text {flat }}$ can be determined in the field, using a pressure-based depth gauge. Then,

$$
\mathbf{q}_{k} \approx\left(q_{x}, q_{y}, z^{\text {flat }}\right)=\mathbf{p}_{k}+\hat{\mathbf{v}}_{\mathrm{w}} z^{\text {flat }} / s_{z}^{\mathrm{w}} .
$$

\subsection{Reconstruction}

Surface estimation from its sampled normals $\left\{\hat{\mathbf{N}}_{k}\right\}$ is closely related to shape from shading [27, 33]. The WAI is typically smooth and integrable. Thus, we perform numerical integration of the WAI gradient field (slopes field), which is derived from $\left\{\hat{\mathbf{N}}_{k}\right\}$. We use the integration method of Ref. [24] to estimate the WAI shape.

An example of full system computer simulation is shown in Fig. 3. An image of the Stella Maris Monastery was captured from the Haifa Bat-Galim beach. The monastery vista is distorted by a wavy WAI: the WAI is simulated as in $[23,46]$ by a wind of $1.5 \mathrm{~m} / \mathrm{s}$, and has peak-to-peak amplitude of $2 \mathrm{~cm}$. Sunrays project onto the WAI, refract, pass the pinhole array and irradiate the diffuser. Using a simulated image of the diffuser, the WAI shape is estimated. The reconstructed WAI is similar to the ground truth, but it exhibits a bias. The unrecoverable bias is explained in Sec. 3.1. Image recovery estimates the viewing elevation and azimuth angles, in air, corresponding to $\hat{\mathbf{v}}_{\mathrm{a}}$, per image pixel. A scatter plot compares view angles recovered based on the estimated wavy WAI, to the angles resulting only from flat-WAI correction. The angular root mean-squared 

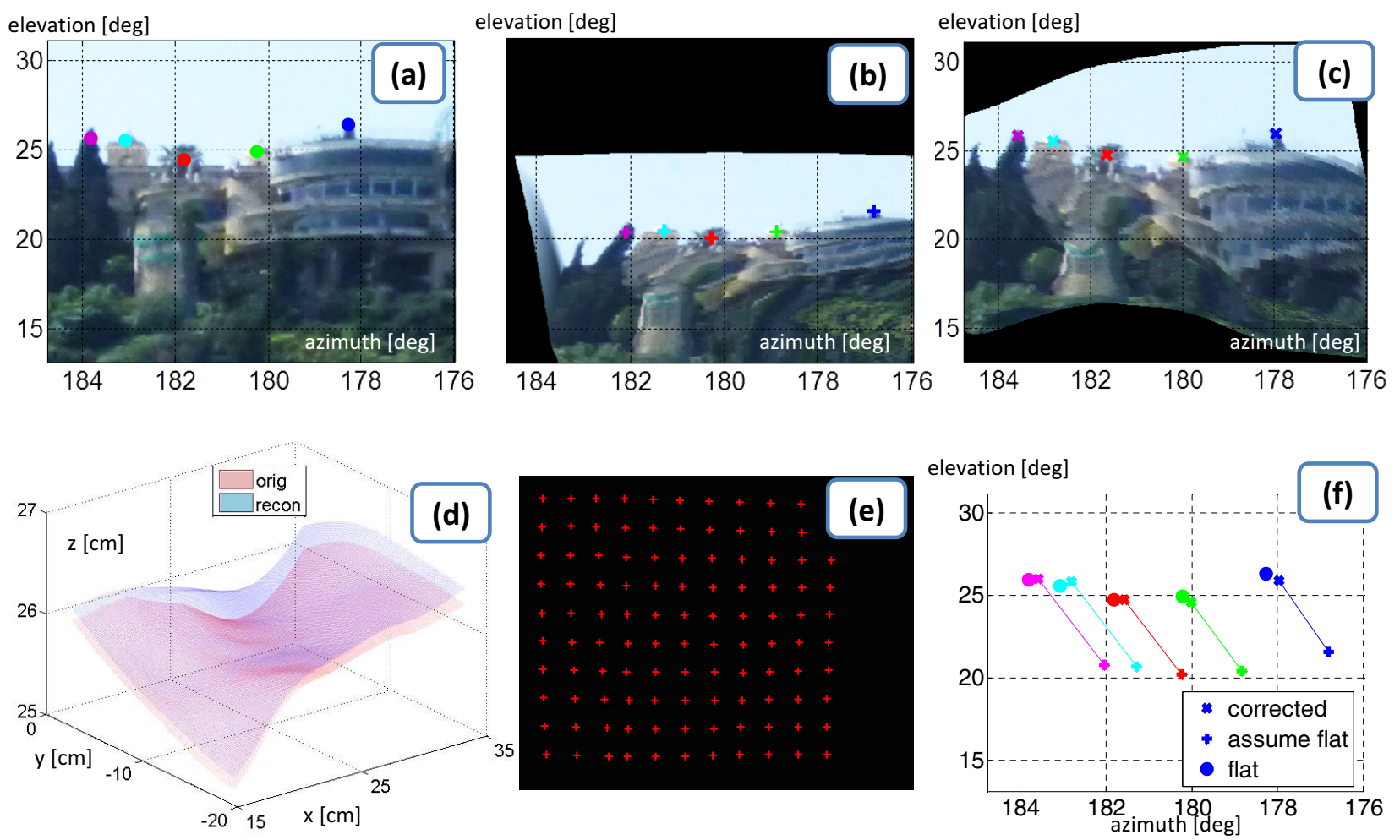

Figure 3. Simulation. (a) Stella Maris Monastery and vista in Haifa, in assigned airborne azimuth and elevation angles. Examined points are overlaid. (b) An image distorted by a wavy WAI. The image is converted by a flat-WAI model to airborne view angle coordinates. The wave in this case shrinks the field of view to a supposedly narrower domain, deflating and distorting objects. (c) Recovered image, in airborne view angle coordinates. (d) Ground-truth WAI [red] and reconstructed WAI [blue]. (e) Locations on the diffuser of sensor $\mathcal{S}$, at which sunrays yield local image maxima. The pattern is quasi-periodic, distorted by the WAI wave. (f) The corresponding color-coded examined points in each image (a-c) are gathered in a scatter plot. It shows significant partial correction of the point locations.

error reduces from $5.04^{\circ}$ to $0.36^{\circ}$, as a result of the wavy WAI estimation. Experiments and an additional simulation are described in Sec. 6.

\section{Limitations}

Some of limitations of a virtual periscope stem [3] from the viewing component $\mathcal{V}$. Limitations of $\mathcal{V}$ are shared by all virtual periscope designs, irrespective of how distortion is estimated. Hence, we focus here on limitations, sensitivities and tradeoffs concerning only the stellar slope-sensor $\mathcal{S}$. Primarily, $\mathcal{S}$ requires sunlight. It is not suitable for overcast days. At night, we believe the principle may be used under moonlight, if lenslets are used in the array, gathering sufficient light power. Other sensitivities and limitations of $\mathcal{S}$ are geometric, as analyzed next.

\subsection{Sensitivity to Variations of $\hat{\mathrm{N}}$}

The WAI normal is perturbed around the $\hat{\mathbf{z}}$ axis. In general, the vector $\hat{\mathbf{s}}^{\mathrm{W}}$ is obtained by Snell's law [16]

$$
\hat{\mathbf{s}}^{\mathrm{w}}=\frac{\hat{\mathbf{s}}^{\mathrm{a}}}{n}-\frac{\hat{\mathbf{N}}}{n}\left[\hat{\mathbf{N}} \cdot \hat{\mathbf{s}}^{\mathrm{a}}-\sqrt{n^{2}-1+\left(\hat{\mathbf{N}} \cdot \hat{\mathbf{s}}^{\mathrm{a}}\right)^{2}}\right] .
$$

Substituting $\hat{\mathbf{N}}=\hat{\mathbf{z}}$ into Eq. (13) yields the in-water sun-ray vector, when the water is flat, $\hat{\mathbf{s}}_{\text {flat }}^{\mathrm{W}}$. Due to the perturbation, the in-water refractive angle changes by

$$
\psi=\arccos \left(\hat{\mathbf{s}}^{\mathrm{w}} \cdot \hat{\mathbf{s}}_{\text {flat }}^{\mathrm{w}}\right) .
$$

The perturbation has two principal components. The

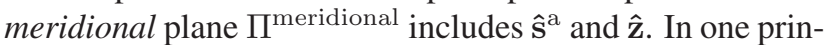
cipal components, the WAI normal $\hat{\mathbf{N}}$ is perturbed within this plane ( $\left.\hat{\mathbf{N}} \| \Pi^{\text {meridional }}\right)$, i.e., $\hat{\mathbf{N}}$ rotates around an axis $\hat{\omega}_{\|}=\hat{\mathbf{s}}^{\mathrm{a}} \times \hat{\mathbf{z}}$. For simplicity of notations, let $\Pi^{\text {meridional }}$ be the $x z$ plane. Hence $\hat{\mathbf{s}}^{\mathrm{a}}=\left(\sin \theta_{\text {sun }}, 0, \cos \theta_{\text {sun }}\right)^{\mathrm{T}}$, where $\theta_{\text {sun }}$ is the solar zenith angle in air. In this case,

$$
\hat{\mathbf{N}}=\hat{\mathbf{N}}_{\text {merdional }}=(\sin \theta, 0, \cos \theta)^{\mathrm{T}},
$$

where $\theta$ is the WAI zenith angle.

In the other perturbation component, $\hat{\mathbf{N}}$ tilts out of $\Pi^{\text {meridional }}$, i.e., $\hat{\mathbf{N}}$ rotates around an axis $\hat{\omega}_{\perp}=\hat{\mathbf{z}} \times\left(\hat{\mathbf{s}}^{\mathrm{a}} \times \hat{\mathbf{z}}\right)$. Now, $\hat{\mathbf{N}}$ resides in the sagittal plane $\Pi^{\text {sagitt }} \perp \Pi^{\text {meridional }}$. If $\Pi^{\text {meridional }}$ is the $x z$ plane, then

$$
\hat{\mathbf{N}}=\hat{\mathbf{N}}_{\text {sagitt }}=(0, \sin \theta, \cos \theta)^{\mathrm{T}} \text {. }
$$


Substituting Eq. (15) or (16) into Eqs. $(13,14)$, derives the ray angular perturbation in water. Sensitivity to perturbations is assessed by $\left.(d \psi / d \theta)\right|_{\theta=0}$. A plot reveals that the sensitivities monotonically increase with $\theta_{\text {sun }}$. As $\theta_{\text {sun }} \rightarrow 90^{\circ}$, i.e., in mornings or evenings, sensitivities are highest: $d \psi / d \theta=1$ for meridional perturbations and $d \psi / d \theta=0.66$ for sagittal. Sensitivity is minimal at $\theta_{\text {sun }}=0^{\circ}$ (tropical noon). There, $d \psi / d \theta=0.25$ in both perturbation components. Sensitivities thus have similar order of magnitude in different geometries.

\subsection{Resolution Tradeoff}

Neighboring pinholes in $\mathcal{S}$ are $D$ apart (Fig. 4). Reducing $D$ enables recovery of shorter WAI wavelengths. However, as we describe below, a shorter $D$ decreases the angular resolution of $\hat{\mathbf{N}}$. The angular resolution of $\hat{\mathbf{N}}$ increases with $z_{h}$, but the angular dynamic range decreases with $z_{h}$. We now quantify these tradeoffs in the meridional $(x z)$ plane, based on geometric optics.

For the moment, assume that the sun is a point source. The $x$ coordinate of pinhole $k$ is $h_{k}$, while $h_{k+1}-h_{k}=D$. The sunray projecting through pinhole $k$ has in-water direction vector $\hat{\mathbf{s}}_{k}^{\mathrm{w}}$, with a corresponding zenith angle

$\theta_{k}^{\mathrm{ws}}=\arccos \left(\hat{\mathbf{s}}_{k}^{\mathrm{w}} \cdot \hat{\mathbf{z}}\right)=\theta_{\text {flat }}^{\mathrm{ws}}+\psi_{k}=\arccos \left(\hat{\mathbf{s}}_{\text {flat }}^{\mathrm{w}} \cdot \hat{\mathbf{z}}\right)+\psi_{k}$.

Here $\psi_{k}$ is the deviation of the refracted solar angle in-water (relative to the angle in flat-water, $\theta_{\text {flat }}^{\text {ws }}$ ). This ray intersects the diffuser at $x$ coordinate

$$
p_{k}=h_{k}-z_{h} \tan \theta_{k}^{\mathrm{ws}} \text {. }
$$

Let the sensor $\mathcal{S}$ determine $p_{k}$ with spatial uncertainty $\Delta p$. This uncertainty may be due to camera pixel size, diffuser

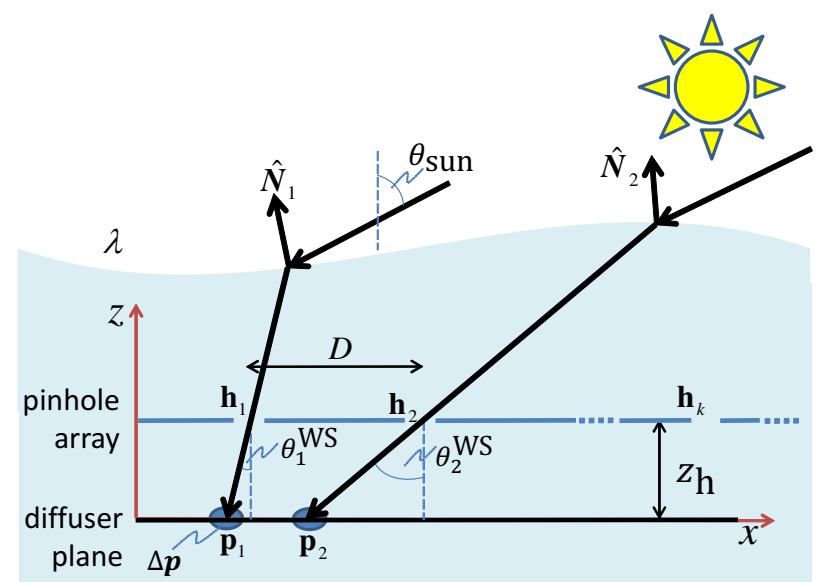

Figure 4 . Geometry of $\mathcal{S}$. Pinhole $k$ at $h_{k}$ yields a stellar image-let at $p_{k}$. It samples normal $\hat{\mathbf{N}}_{k}$ of a WAI having wavelength $\lambda$. The corresponding sun-ray angle is $\theta_{k}^{\text {ws }}$ in water and $\theta_{\text {sun }}$ in air. Measurement of $p_{k}$ by $\mathcal{S}$ has uncertainty $\Delta p$. Pinholes are at height $z_{h}$ above a diffuser. characteristics or pinhole size. This spatial uncertainty converts to uncertainties in the measured angular deviation $\Delta \psi$ and the WAI-normal angle $\Delta \theta$

$$
\Delta p=\frac{z_{h}}{\cos ^{2} \theta^{\mathrm{ws}}} \Delta \psi \approx \frac{z_{h}}{\cos ^{2} \theta_{\text {flat }}^{\mathrm{ws}}} \frac{d \psi}{d \theta} \Delta \theta
$$

Given $\Delta p$, a longer $z_{h}$ refines the discrimination $\Delta \theta$.

When the WAI is flat, it is simple to establish correspondence of $p_{k}$ with $h_{k}$, since $p_{k}<p_{k+1}$, similarly to the ordering of $h$. To maintain correct correspondence, this relation has to be satisfied also when the WAI is wavy, $\forall k$ :

$$
p_{k}+\Delta p / 2<p_{k+1} \text {. }
$$

Eq. (20) includes the small margin $\Delta p / 2$, so that adjacent solar projections $p_{k+1}, p_{k}$ do not merge to a single spot. From Eqs. $(17,18,20)$,

$$
\begin{aligned}
D-\Delta p / 2 & >z_{h}\left(\tan \theta_{k+1}^{\mathrm{ws}}-\tan \theta_{k}^{\mathrm{ws}}\right) \\
& \approx \frac{z_{h}}{\cos ^{2} \theta_{\text {flat }}^{\mathrm{ws}}}\left(\psi_{k+1}-\psi_{k}\right) \\
& \approx \frac{z_{h}}{\cos ^{2} \theta_{\text {flat }}^{\mathrm{ws}}} \frac{d \psi}{d \theta}\left(\theta_{k+1}-\theta_{k}\right)
\end{aligned}
$$

Hence, a too large $z_{h}$ or too small $D$ limits the dynamic range of WAI slope-changes $\left[\theta_{k+1}-\theta_{k}\right]$, beyond which correspondence is erroneous.

Consider a WAI having spatial period $\lambda$, in which the surface angle varies as $\theta(x)=\Theta \cos (2 \pi x / \lambda)$, and $\Theta>0$ is small (WAI slopes are small). In the worst-case, at which $\left(p_{k+1}-p_{k}\right)$ is minimal, the WAI angle changes maximally between samples: $\left|\theta_{k+1}-\theta_{k}\right|=2 \Theta$. To avoid correspondence errors in this scenario,

$$
D>\Delta p / 2+\frac{z_{h}}{\cos ^{2} \theta_{\text {flat }}^{\mathrm{ws}}} \frac{d \psi}{d \theta} 2 \Theta \approx \Delta p / 2+2 \Theta \Delta p / \Delta \theta,
$$

where we used Eq. (19) in (21). As said in Sec. 3.2, the WAI is sampled quasi-periodically. The sampling approaches periodicity of $D$ as $z^{\text {flat }}$ decreases. Thus, we use the Nyquist sampling criterion $(\lambda / 2)>D$ for avoiding aliasing. Compounding it with Eq. (22) yields

$$
\lambda>\frac{\Delta p}{2} \frac{\Theta}{\Delta \theta}(8+\Delta \theta / \Theta)
$$

In Eq. (23), the fraction $\Delta \theta / \Theta$ expresses the relative angular resolution in which the WAI can be recovered: the ratio of uncertainty to dynamic range. Since $\Delta \theta / \Theta \ll 8$, we obtain an uncertainty principle

$$
\frac{\Delta \theta}{\Theta} \cdot \lambda>4 \Delta p
$$




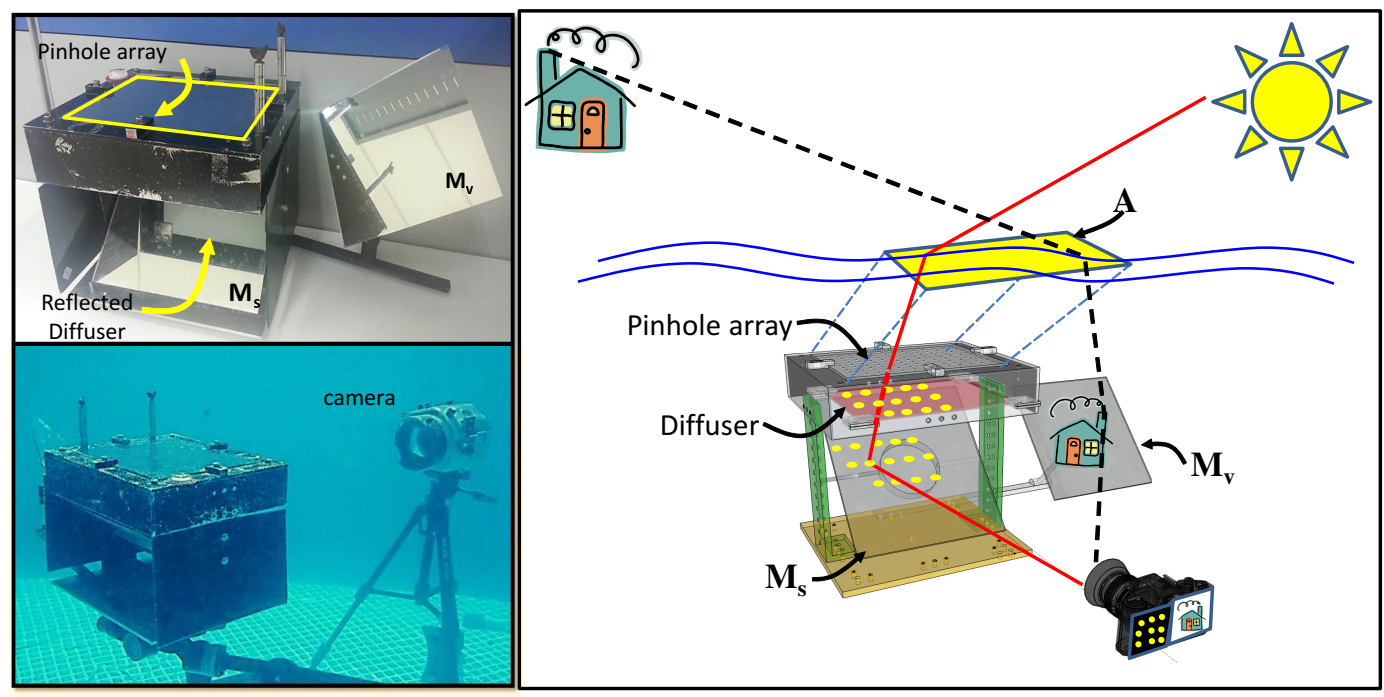

Figure 5. [Left] Hardware implementation. [Right] observing through WAI area $\mathcal{A}$, which is estimated based on sun-imaging.

The relative WAI slope angular resolution can be traded-off for spatial resolution $(\lambda)$ of the WAI, before errors stemming from aliasing and correspondence take effect.

Despite all efforts, errors are made. Some waves contain significant energy in high spatial frequencies, leading to aliasing, or, their slope variations are too strong, misleading correspondence (invalidating Eqs. 20,21). Hence, $\mathcal{S}$, on its own, does not enable complete compensation of the distortions observed by $\mathcal{V}$, in all frames and pixels. However, $\mathcal{S}$ enables significant reduction of the distortion.

\section{Parallel Sunrays?}

Two effects deviate from the parallel sunrays model, leading to broadening of $\Delta p$ with $z_{\mathrm{h}}$. First, the Sun occupies a finite solid angle in the sky, causing sunray spread over a range of angles in air, and thus in $\mathcal{S}$. In our system implementation, (Secs. 5,6), this angular spread translates to lateral blur of $0.2 \mathrm{~mm}$ on the diffuser plane, significantly smaller than $\Delta p \approx 2 \mathrm{~mm}$ of our overall system. Second, light-wave diffraction through a pinhole does not obey a ray model. Our pinholes are $0.8 \mathrm{~mm}$ wide. For visible light (green) and our implementation, diffraction spreads the projected Sun image by $2 \mu \mathrm{m} \ll \Delta p$. Hence, both effects are insignificant in our design. However, in other designs they can be more significant, changing the tradeoff relations.

\section{Sensor Design}

Components $\mathcal{S}$ and $\mathcal{V}$ must image their respective fields simultaneously. This can be achieved by careful mutual synchronization of two cameras, one per component. We ensure synchronization in a different, simple design. We use $a$ single camera to simultaneously image both the diffuser in $\mathcal{S}$ and the field of $\mathcal{V}$. We were inspired by designs using a single camera and mirrors for synchronized multiview images $[10,20]$, at a cost of spatial resolution. Our design is depicted in Fig. 5. The camera field of view has two main parts. One part is dedicated to image the diffuser of $\mathcal{S}$, through mirror $M_{\mathcal{S}}$. The other part views the airborne scene of interest, through a view mirror $M_{\mathcal{V}}$. The WAI region $\mathcal{A}$ that is reconstructed using $\mathcal{S}$ is generally laterally offset from $\mathcal{S}$, according to $\hat{\mathrm{s}}^{\mathrm{a}}$. The airborne scene must be imaged through $\mathcal{A}$. Thus, $M_{\mathcal{V}}$ is adjusted accordingly.

\section{Results}

In our implementation, the pinhole array was a thin metal sheet manufactured using precise laser cutting. It was laid on a glass plate. The diffuser was made of opal glass. The camera was a Nikon D7100. System calibration is described in [3]. Extracting the projected solar locations on the diffuser involves a series of simple image processing steps, illustrated in Fig. 6. In some experiments the camera was separated from the water by a flat-glass interface. The system, thus, has a non-single viewpoint [49]. However, our fields of view (per $\mathcal{S}$ and $\mathcal{V}$ ) were small. Hence, as we verified, the single viewpoint approximation was sufficient. System leveling was verified by a leveler. We had $D=1.8 \mathrm{~cm}$, $z_{h}=6.18 \mathrm{~cm}$ and $z^{\text {flat }}=25.7 \mathrm{~cm}$. Exposure time was $4 \mathrm{~ms}$. System azimuth was measured by a compass.

Before experiments, we made simulations based on the system parameters. One simulation is described in Sec. 3.3 and Fig. 3. Another simulation, based on similar wind and solar conditions, imaged a checkerboard at height $z_{a}=1 \mathrm{~m}$. The results are shown in Fig. 7. A scatter plot expresses the width and length $\left(l_{x}\right.$ and $\left.l_{y}\right)$ of the square checkers, in three cases: ground-truth, $2.1 \mathrm{~cm}$ (blue); recovery resulting only from flat-WAI correction (red); and recovery based on the 


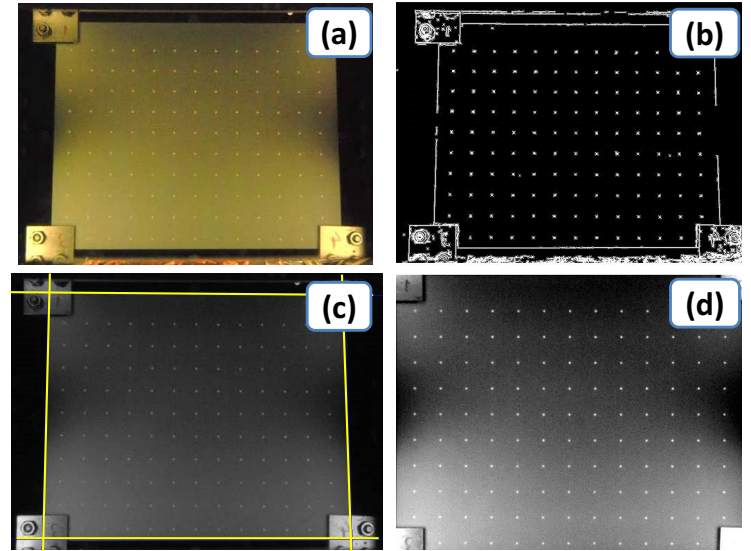

Figure 6. Processing image (a) of $\mathcal{S}$ : (b) Edge detection followed by Hough transforms, lead to the diffuser corners (c). Rectifying the image, such that the diffuser corners span a $25 \times 20 \mathrm{~cm}^{2}$ rectangle (d), over a regular pixel grid. Fitting Gaussians to local maxima in (d) localizes the solar projections.
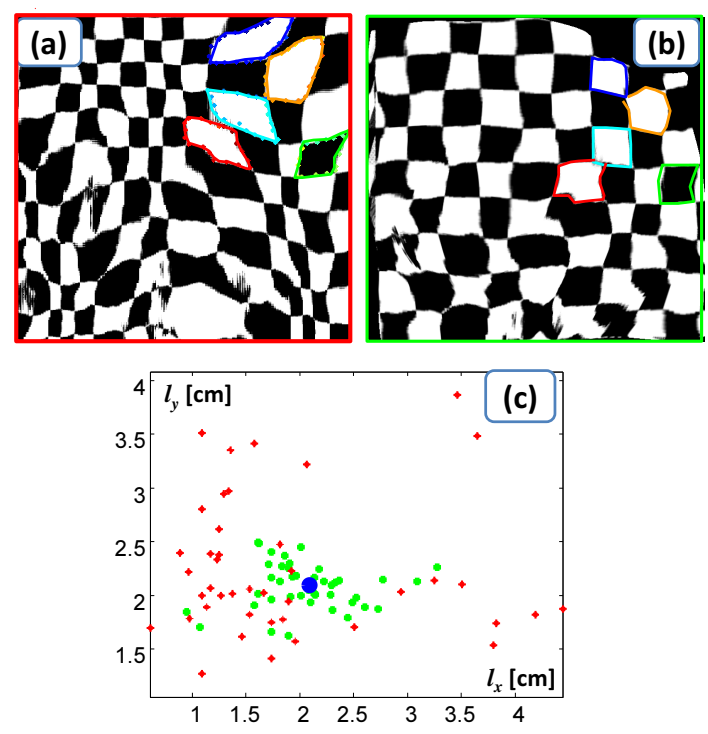

Figure 7. (a) A simulated distorted image. (b) An image corrected using the estimated wavy WAI. (c) A scatter plot showing widths and lengths $\left(l_{x}\right.$ and $\left.l_{y}\right)$ of the square checkers, in three cases: ground-truth, $2.1 \mathrm{~cm}$ (blue); estimation based on the estimated wavy WAI (green) or only on flat-WAI correction (red).

estimated wavy WAI (green). When only flat-WAI correction is applied, the size standard deviation (STD) of squares in the distorted pattern is $1.68 \mathrm{~cm}$. The STD decreases to $0.33 \mathrm{~cm}$, as a result of the wavy WAI estimation.

We tested this calibrated scenario in a real experiment by our system. The checkerboard laid above the water at $z_{\mathrm{a}}=118 \mathrm{~cm}$. The sun zenith angle was $\theta_{\text {sun }}=45^{\circ}$. Its azimuth relative to the system's $x$ axis was $-13.5^{\circ}$. The automatically detected spot locations $\left\{\mathbf{p}_{k}\right\}$ and estimated WAI are shown in Fig. 8. Compensation for distortions caused by the wavy WAI (as if $\mathcal{V}$ is in air) yields results shown in Fig. 9. We follow lines passing through adjacent corners of the squares, and then we derive curvatures of these lines. Correction for the waves by our method significantly lowers the mean curvature from $9.8 \cdot 10^{-4}$ to $3.3 \cdot 10^{-4}$. The checkers are significantly more uniform, following wavecorrection. A scatter plot in Fig. 9 expresses the sizes of the square checkers. When size is based only on flat-WAI correction, the size standard deviation (STD) of the squares is $3.13 \mathrm{~cm}$. The STD decreases to $1.3 \mathrm{~cm}$, as a result of the wavy WAI estimation.

We observed a building outdoors. A mirror brought the outdoors view to the water tank lab. In this experiment the sensor had $D=1.2 \mathrm{~cm}$ and $z_{\mathrm{h}}=3.67 \mathrm{~cm}$. We localize seven corners in the building, in 32 consecutive frames. The results are shown in a scatter plot in Fig. 10. Per object point, the airborne direction angles spread wide (crosses), in the absence of correction for the WAI waves. The spread narrows following our process (bold squares). Without our process, the angular STDs are $0.7^{\circ}$ and $0.6^{\circ}$, respectively in azimuth and elevation. The corresponding STDs reduce to $0.4^{\circ}$ and $0.27^{\circ}$, using our system.

We made a sea test session, near Dado Beach (Haifa), illustrated in Fig. 11. Compensation assuming solely flatwater distortion is compared to recovery based on the wave sensor. Compensation relied on knowledge that a person was standing at horizontal distance of $75 \mathrm{~cm}$ from the system. Fig. 12 shows a failure case from the preliminary oceanic experiment. Clearly, during image capture cor-
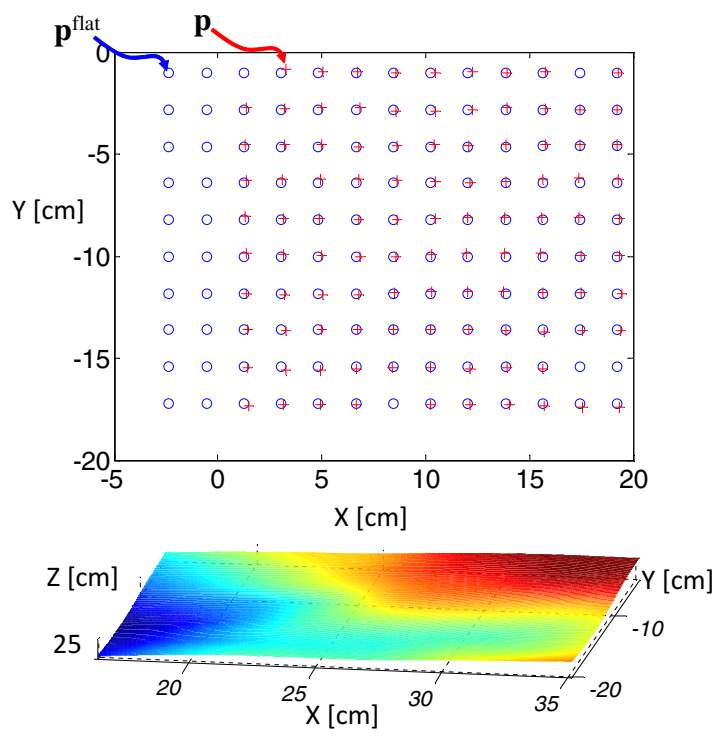

Figure 8. Empirical experiment. [Top] Red crosses are locations where sun image-lets are detected on the diffuser. Blue circles are $\mathbf{p}_{\text {flat }}$, under flat water. [Bottom] Estimated water surface. 


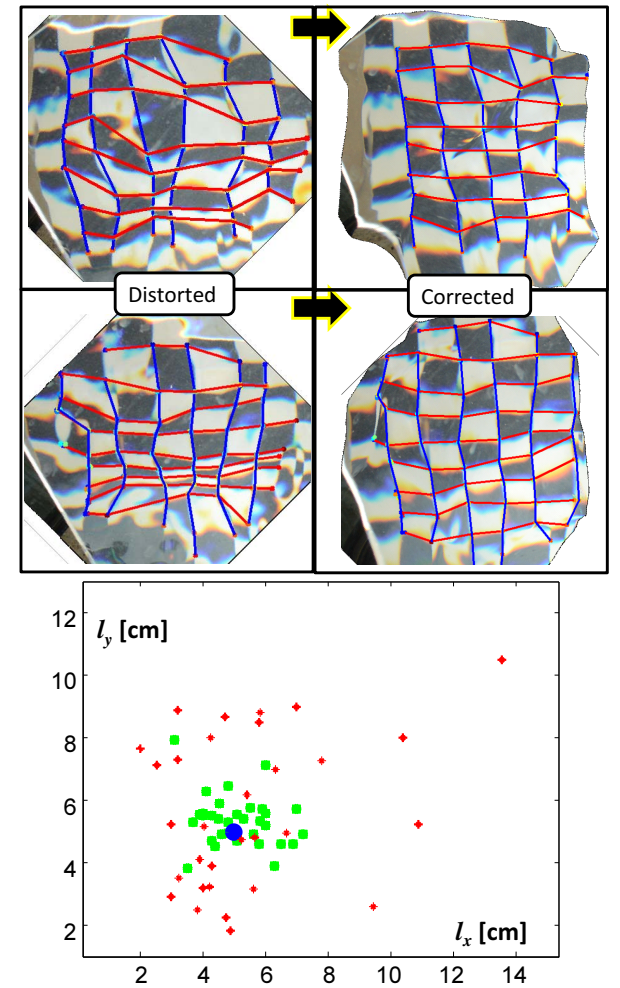

Figure 9. Experiment results. [Top] Distorted and corrected images of the checkerboard. [Bottom] A scatter plot shows the width and length $\left(l_{x}\right.$ and $\left.l_{y}\right)$ of the square checkers on the object: groundtruth, $5 \mathrm{~cm}$ (blue); estimation based on the estimated wavy WAI (green) or only on flat-WAI correction (red).

responding to Fig. 12, the WAI experienced small scale wave ripples. Our existing implementation does not sample the WAI densely enough to measure such high frequency waves, yielding WAI aliasing.

\section{Discussion}

STELLA MARIS is a novel approach to a virtual periscope. It passively measures the water-waves by imaging the refracted Sun (our guide star). Then, the recovered water surface is used to compensate distortions of a scene of interest, captured simultaneously. Distortions can be reduced significantly using this physics-based method, but uncertainties pointed theoretically leave residual distortions. Nevertheless, images corrected by our process can later be handled by statistical un-distortion post-processes [26, 29, 47, 48, 50], such as lucky imaging $[8,9,44]$, stochastic triangulation [2], and motion detection [1]. Rather than facing the full-blown distortions in raw images, such video post-processing methods may handle more easily images whose distortions are residual. Such submerged systems on a sea floor might be useful in biology research. They can monitor habits of seabirds: their flights

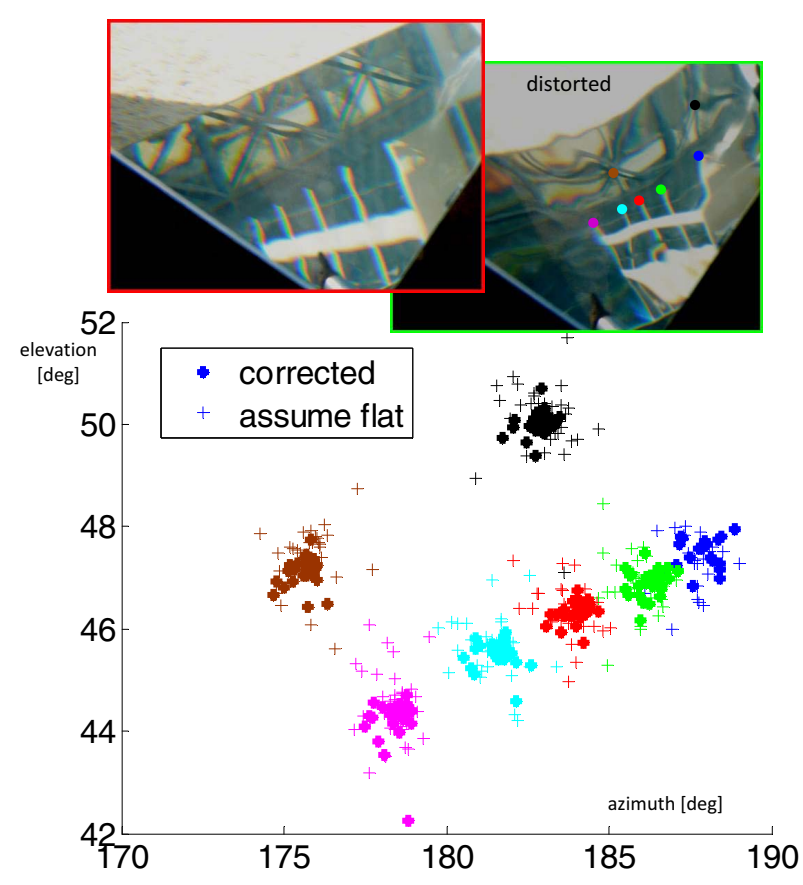

Figure 10. Distant object viewing direction. The examined points of the building are shown at the top. [Bottom] Scatter plot of seven examined points in the viewing direction coordinates. The corrected points are marked as bold dots. The uncorrected points are marked by crosses.

in air, plunge into water and swim for prey.

There are many ways to advance this concept. Using a lenslet array in $\mathcal{S}$ instead of pinholes would increase efficiency. The diffuser can be replaced by other, more efficient optical relay setups. To break the correspondence constraint (20), it is possible to encode pinholes/lenslets $[51,53]$. Sensor $\mathcal{S}$ can be tilted towards the obliquely-illuminating Sun. Taking the analogy to groundbased astronomy even further, we envision an adaptive optics real-time system for our settings. In response to the measured WAI, the distortion in $\mathcal{V}$ can be counter-acted using a flexible-mirror [17].

\section{Acknowledgments}

We thank the anonymous reviewers for their useful comments and Joseph Shamir for useful discussions. We thank Vadim Holodovsky, Amit Aides, Aviad Levis, Yahel David, Guy Nir, Roy Or-El, Tali Treibitz, Danny Veikherman and Daniel Yagodin for helping with the experiments. We thank Zohar Bakfar B\&B for enabling experiments in their swimming pool. Yoav Schechner is a Landau Fellow - supported by the Taub Foundation. The work was supported by the Israel Science Foundation (ISF Grant 1467/12). This work was conducted in the Ollendorff Minerva Center. Minerva is funded through the BMBF. 

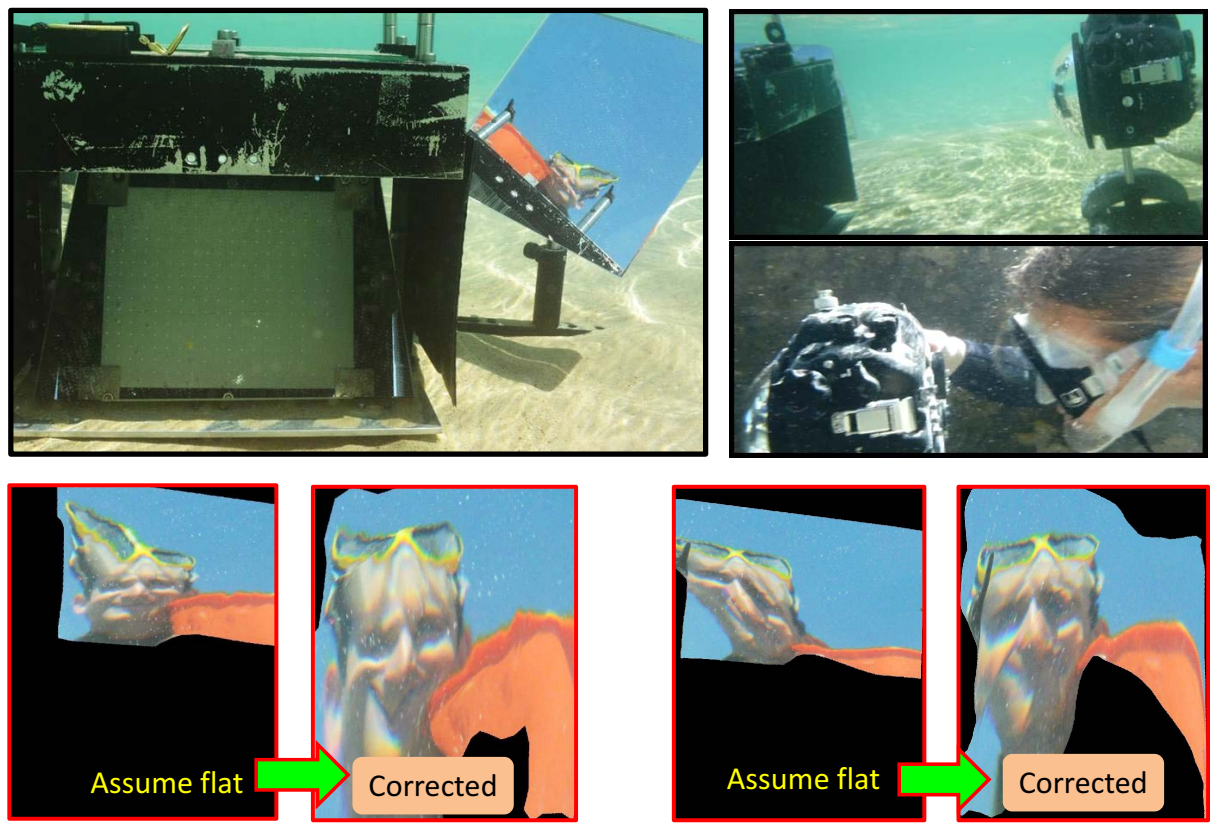

Figure 11. Oceanic experiment. [Top] Experimental setup. [Bottom] Analysis of two frames: flat-water assumption compared to our recovery.

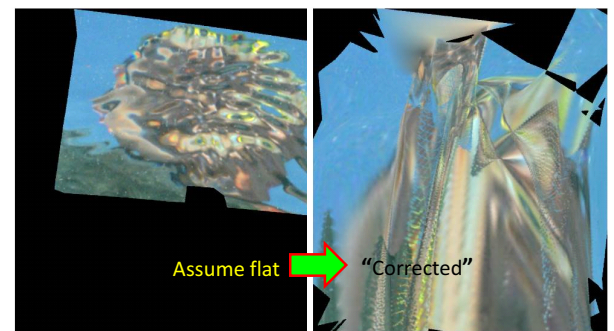

Figure 12. A case of failed un-distortion of a face, in an oceanic experiment.

\section{References}

[1] M. Alterman, Y. Y. Schechner, J. Shamir, and P. Perona. Detecting motion through dynamic refraction. IEEE TPAMI, 35:245-251, 2013.

[2] M. Alterman, Y. Y. Schechner, and Y. Swirski. Triangulation in random refractive distortions. In IEEE ICCP. IEEE, 2013.

[3] M. Alterman, Y. Swirski, and Y. Y. Schechner. STELLA MARIS: Stellar marine refractive imaging sensor - supplemental material - calibration. IEEE ICCP, 2014.

[4] A. Ashok and M. A. Neifeld. Compressive light field imaging. In SPIE Defense, Security, and Sensing, 2010.

[5] T. Bishop and P. Favaro. The light field camera: Extended depth of field, aliasing, and superresolution. IEEE TPAMI, 34(5):972-986, 2012.

[6] P. Cobelli, A. Maurel, V. Pagneux, and P. Petitjeans. Global measurement of water waves by Fourier transform profilometry. Exper. in Fluids, 46:1037-1047, 2009.
[7] Y. Ding, F. Li, Y. Ji, and J. Yu. Dynamic 3D fluid surface acquisition using a camera array. IEEE ICCV, 2011.

[8] A. Donate and E. Ribeiro. Improved reconstruction of images distorted by water waves. Adv. Comp. Graph. \& Comp. Vision, 264-277, 2007.

[9] A. Efros, V. Isler, J. Shi, and M. Visontai. Seeing through water. NIPS, 17:393-400, 2004.

[10] J. Gluckman and S. Nayar. Catadioptric Stereo Using Planar Mirrors. IJCV, 44(1):65-79, 2001.

[11] W. Heidrich, H. Lensch, M. F. Cohen, and H.-P. Seidel. Light field techniques for reflections and refractions. In Eurographics conf. Rendering, 187-196, 1999.

[12] I. Ihrke, B. Goidluecke, and M. Magnor. Reconstructing the geometry of flowing water. In IEEE ICCV, volume 2, pages 1055-1060, 2005.

[13] I. Ihrke, K. Kutulakos, H. P. Lensch, M. A. Magnor, and W. Heidrich. State of the art in transparent and specular object reconstruction. Proc. Eurographics, 2008.

[14] B. Jähne, J. Klinke, and S. Waas. Imaging of short ocean wind waves: a critical theoretical review. JOSA, 11:21972209, 1994.

[15] N. Joshi and M. F. Cohen. Seeing Mt. Rainier: Lucky imaging for multi-image denoising, sharpening, and haze removal. In ICCP, 2010.

[16] M. J. Kidger. Fundamental Optical Design. SPIE Press, 2002.

[17] S. Kuthirummal and S. K. Nayar. Flexible mirror imaging. In IEEE ICCV, 2007.

[18] I. M. Levin, V. V. Savchenko, and V. J. Osadchy. Correction of an image distorted by a wavy water surface: laboratory experiment. Appl. Opt., 47:6650-6655, 2008. 
[19] A. Lumsdaine and T. Georgiev. The focused plenoptic camera. In IEEE ICCP, pages 1-8, 2009.

[20] A. Manakov, J. F. Restrepo, O. Klehm, R. Hegedüs, E. Eisemann, H.-P. Seidel, and I. Ihrke. A reconfigurable camera add-on for high dynamic range, multi-spectral, polarization, and light-field imaging. SIGGRAPH, 32(4):47, 2013.

[21] M. Micheli, Y. Lou, S. Soatto, and A. Bertozzi. A linear systems approach to imaging through turbulence. J. Math. Imaging and Vision, 2013.

[22] D. M. Milder, P. W. Carter, N. L. Flacco, B. E. Hubbard, N. M. Jones, K. R. Panici, B. D. Platt, R. E. Potter, K. W. Tong, and D. J. Twisselmann. Reconstruction of throughsurface underwater imagery. Waves in Random and Complex Media, 16:521 - 530, 2006.

[23] J. Miranda, A. Camps, J. Gomez, M. Vall-llossera, and R. Villarino. Time-dependent sea surface numerical generation for remote sensing applications. In Proc. IGARSS, pages $2527-2530,2005$.

[24] F. Moisy, M. Rabaud, and K. Salsac. A synthetic Schlieren method for the measurement of the topography of a liquid interface. Exper. in fluids, 46(6):1021-1036, 2009.

[25] N. Morris and K. Kutulakos. Dynamic refraction stereo. In IEEE ICCV, volume 2, pages 1573-1580, 2005.

[26] H. Murase. Surface shape reconstruction of a nonrigid transport object using refraction and motion. IEEE TPAMI, 14:1045-1052, 1992.

[27] S. G. Narasimhan, S. K. Nayar, B. Sun, and S. J. Koppal. Structured light in scattering media. In IEEE ICCV, volume 1, pages 420-427, 2005.

[28] R. Ng, M. Levoy, M. Brédif, G. Duval, M. Horowitz, and P. Hanrahan. Light field photography with a hand-held plenoptic camera. CS Tech. Rep. CSTR, 2(11), 2005.

[29] O. Oreifej, G. Shu, T. Pace, and M. Shah. A two-stage reconstruction approach for seeing through water. IEEE CVPR, 2011.

[30] E. N. Ribak, E. Gershnik, and M. Cheselka. Stellar scintillations as a remote atmospheric wave-front sensor. Opt. Lett., 21(6):435-437, 1996.

[31] T. Roesgen, A. Lang, and M. Gharib. Fluid surface imaging using microlens arrays. Exper. in Fluids, 25:126-132, 1998.

[32] M. Roggemann and B. Welsh. Imaging through turbulence. CRC press, 1996.

[33] I. Sato, T. Okabe, and Y. Sato. Bispectral photometric stereo based on fluorescence. In IEEE CVPR, pages 270-277, 2012.

[34] R. Savelsberg, A. Holten, and W. van de Water. Measurement of the gradient field of a turbulent free surface. Exper. in Fluids, 41:629-640, 2006.

[35] Y. Y. Schechner. A view through the waves. Marine Technology Society Journal, 47:148-150, 2013.

[36] Y. Y. Schechner and N. Karpel. Attenuating natural flicker patterns. In MTS/IEEE Oceans, pages 1262-1268, 2004.

[37] Y. Y. Schechner, J. Shamir, and N. Kiryati. Polarization and statistical analysis of scenes containing a semi-reflector. $J$. Opt. Soc. Am. A, 17(2):276-284, 2000.

[38] M. S. Schmalz. Integration of stereophotogrammetry with image restoration models for distortion-tolerant viewing through the sea surface. Proc. SPIE, 1943:115-128, 1993.
[39] H. Schultz. Shape reconstruction from multiple images of the ocean surface. Photogrammetric eng. and remote sensing, 62(1):93-101, 1996.

[40] H. Schultz and A. Corrada-Emmanuel. System and method for imaging through an irregular water surface, 2007. US Patent 7,630,077.

[41] F. J. Serón, D. Gutierrez, G. Gutierrez, and E. Cerezo. Implementation of a method of curved ray tracing for inhomogeneous atmospheres. Com. \& Graph.,95-108, 29, 2005.

[42] J. Shamir, D. G. Crowe, and J. W. Beletic. Improved compensation of atmospheric turbulence effects by multiple adaptive mirror systems. Appl. Opt., 32(24):4618-4628, 1993.

[43] A. Stern and B. Javidi. Three-dimensional image sensing, visualization, and processing using integral imaging. IEEE, 94:591-607, 2006

[44] H. Suiter, N. Flacco, P. Carter, K. Tong, R. Ries, and M. Gershenson. Optics Near the Snell Angle in a Water-to-Air Change of Medium. In IEEE OCEANS, 2008.

[45] Y. Swirski and Y. Y. Schechner. 3Deflicker from motion. In IEEE ICCP, 2013.

[46] Y. Swirski, Y. Y. Schechner, B. Herzberg, and S. Negahdaripour. CauStereo: Range from light in nature. App. Opt., 50:89-101, 2011.

[47] Y. Tian and S. G. Narasimhan. Seeing through water: Image restoration using model-based tracking. In IEEE ICCV, 2303-2310, 2009.

[48] Y. Tian and S. G. Narasimhan. A globally optimal datadriven approach for image distortion estimation. IEEE CVPR, 2010.

[49] T. Treibitz, Y. Y. Schechner, C. Kunz, and H. Singh. Flat refractive geometry. IEEE Tran. on PAMI, 34(1):51-65, 2012.

[50] Z. Wen, A. Lambert, D. Fraser, and H. Li. Bispectral analysis and recovery of images distorted by a moving water surface. In JOSA A, volume 49, pages 6376-6384, 2010.

[51] G. Wetzstein, D. Roodnick, W. Heidrich, and R. Raskar. Refractive shape from light field distortion. In IEEE ICCV, 1180 $-1186,2011$.

[52] J. Ye, Y. Ji, F. Li, and J. Yu. Angular domain reconstruction of dynamic $3 \mathrm{~d}$ fluid surfaces. In IEEE CVPR, pages 310$317,2012$.

[53] X. Zhang and C. S. Cox. Measuring the two-dimensional structure of a wavy water surface optically: A surface gradient detector. Exper. in Fluids, 17:225-237, 1994.

[54] X. Zhu and P. Milanfar. Removing atmospheric turbulence via space-invariant deconvolution. IEEE TPAMI, 35(1):157 $-170,2013$. 